\title{
On the choice of the smoothing parameter for the BHEP goodness-of-fit test*
}

\author{
Carlos Tenreiro ${ }^{\dagger}$
}

November 22, 2008

\begin{abstract}
The BHEP (Baringhaus-Henze-Epps-Pulley) test for assessing univariate and multivariate normality has shown itself to be a relevant test procedure, recommended in some recent comparative studies. It is well known that the finite sample behaviour of the BHEP goodness-of-fit test strongly depends on the choice of a smoothing parameter $h$. A theoretical and finite sample based description of the role played by the smoothing parameter in the detection of departures from the null hypothesis of normality is given. Additionally, the results of a Monte Carlo study are reported in order to propose an easy-to-use rule for choosing $h$. In the important multivariate case, and contrary to the usual choice of $h$, the BHEP test with the proposed smoothing parameter presents a comparatively good performance against a wide range of alternative distributions. In practice, if no relevant information about the tail of the alternatives is available, the use of this new bandwidth is strongly recommended. Otherwise, new choices of $h$ which are suitable for short tailed and long tailed alternative distributions are also proposed.
\end{abstract}

KEYWORDs: BHEP goodness-of-fit test, kernel density estimator, Bahadur efficiency, multivariate normality, Monte Carlo power comparison.

AMS 2000 SUBJECT CLASSifiCATIONS: 62G10, 62G20.

*This is an electronic version of an article published in Computational Statistics and Data Analysis (Vol. 53, 2009, 1038-1053), and available on line at http://dx.doi.org/10.1016/j.csda.2008.09.002

${ }^{\dagger}$ CMUC, Department of Mathematics, University of Coimbra, Apartado 3008, 3001-454 Coimbra, Portugal. E-mail: tenreiro@mat.uc.pt. URL: http://www.mat.uc.pt/ tenreiro/. 


\section{Introduction}

Let $X_{1}, X_{2}, \ldots, X_{n}, \ldots$ be a sequence of independent and identically distributed $d$-dimensional random vectors with unknown density function $f$. Following an idea of Anderson et al. [1] that used kernel density estimators with fixed bandwidths for testing the equality of two multivariate probability density functions, Fan [17] uses the Bickel-Rosenblatt statistic (cf. Bickel and Rosenblatt [10]; see also Fan [16] and Gouriéroux and Tenreiro [20]) with a constant bandwidth for testing the composite hypothesis that $f$ is a member of a general parametric family of density functions. The test statistic is based on the $L_{2}$ distance between the kernel density estimator and an estimator of its mathematical expectation under the null hypothesis and it is given by

$$
I_{n}^{2}(h)=n \int\left\{f_{n}(x)-\overline{\mathrm{E}}_{0} f_{n}(x)\right\}^{2} d x
$$

where the unspecified integral denotes integration over the whole space,

$$
f_{n}(x)=\frac{1}{n} \sum_{i=1}^{n} K_{h}\left(x-X_{i}\right),
$$

for $x \in \mathbb{R}^{d}, K_{h}(\cdot)=K(\cdot / h) / h^{d}$ with $K$ a kernel, that is, a bounded and integrable function on $\mathbb{R}^{d}$, and $h$ is a strictly positive real number.

In this paper our attention will be focused on the important case where the null parametric family of density functions is the Gaussian family

$$
g(\cdot ; m, S)=\left|S^{-1 / 2}\right| \phi\left(S^{-1 / 2}(\cdot-m)\right),
$$

with $\phi$ the $d$-dimensional standard Gaussian density

$$
\phi(x)=(2 \pi)^{-d / 2} \exp \left(-\frac{1}{2} x^{\prime} x\right)
$$

for $x \in \mathbb{R}^{d}$, where the prime denotes transpose. Although an extensive literature exists regarding the problem of testing for univariate and multivariate normality, there is a continued interest in this classical problem as attested by the recent papers of Mecklin and Mundfrom [28], Arcones [2], Arcones and Wang [3], Farrel et al. [19], Arcones [4], Coin [12] and Yazici and Yolacan [35]. In the case of testing a multivariate hypothesis of normality, a location-scale invariant version of the previous statistic with $K$ the standard Gaussian density, was first considered by Bowman and Foster [11] (with $h \rightarrow 0$ ). Denoting by

$$
\bar{X}_{n}=\frac{1}{n} \sum_{j=1}^{n} X_{j}
$$


the sample mean vector and by

$$
S_{n}=\frac{1}{n} \sum_{j=1}^{n}\left(X_{j}-\bar{X}_{n}\right)\left(X_{j}-\bar{X}_{n}\right)^{\prime},
$$

the sample covariance matrix, the location-scale invariant version of $I_{n}^{2}(h)$ is given by

$$
\mathbf{I}_{n}^{2}(h)=n\left|S_{n}^{1 / 2}\right| \int\left\{\bar{f}_{n}(x)-\varphi_{n}\left(x ; \bar{X}_{n}, S_{n}\right)\right\}^{2} d x
$$

where, for $x \in \mathbb{R}^{d}$,

$$
\begin{gathered}
\bar{f}_{n}(x)=\frac{1}{n} \sum_{i=1}^{n} K_{H_{n}}\left(x-X_{i}\right), \\
K_{H_{n}}(\cdot)=\left|H_{n}^{-1}\right| \phi\left(H_{n}^{-1} \cdot\right), \\
H_{n}=h S_{n}^{1 / 2},
\end{gathered}
$$

with $h>0, S_{n}^{1 / 2}$ the square root of $S_{n}$ and, for $m \in \mathbb{R}^{d}$ and $S$ a non-singular covariance matrix,

$$
\varphi_{n}(x ; m, S)=\int K_{H_{n}}(x-y) g(y ; m, S) d y .
$$

A unifying study of the asymptotic behaviour of statistic (3) for the non-fixed $(h \rightarrow 0)$ and fixed $(h>0)$ bandwidth cases is presented in Tenreiro [34]. The statistic (3) is particularly interesting because the calculation of $\mathbf{I}_{n}^{2}(h)$ does not require any integration (cf. Bowman and Foster [11] and Henze [24]):

$$
\mathbf{I}_{n}^{2}(h)=\frac{1}{n} \sum_{i, j=1}^{n} \mathbf{Q}\left(Y_{i}, Y_{j} ; h\right),
$$

with

$$
Y_{i}=S_{n}^{-1 / 2}\left(X_{i}-\bar{X}_{n}\right)
$$

and

$$
\mathbf{Q}(u, v ; h)=\phi_{\left(2 h^{2}\right)^{1 / 2}}(u-v)-\phi_{\left(1+2 h^{2}\right)^{1 / 2}}(u)-\phi_{\left(1+2 h^{2}\right)^{1 / 2}}(v)+\phi_{\left(2+2 h^{2}\right)^{1 / 2}}(0),
$$

for $u, v \in \mathbb{R}^{d}$.

Apparently in an independent way, the statistic $\mathbf{I}_{n}^{2}(h)$ has been previously considered by several authors and is known in the literature as the BHEP (Baringhaus-Henze-EppsPulley) statistic where the smoothing parameter is usually denoted by $\beta$ and is connected with $h$ through the relation $h=1 /(\beta \sqrt{2}$ ) (see Henze [24]). It has been introduced in the univariate case by Epps and Pulley [15] and in the multivariate case by Baringhaus 
and Henze [8] and Henze and Zirkler [23] as an empirical characteristic function based goodness-of-fit test. In fact, similarly to what was pointed out by Fan [17] in relation to statistic (1), the statistic $\mathbf{I}_{n}^{2}(h)$ can be interpreted as a $L_{2}$ weighted distance between the empirical characteristic function and the parametric estimate of the characteristic function implied by the null model with weight function $t \rightarrow|\hat{\phi}(t h)|^{2}$, where $\hat{\phi}$ is the characteristic function of $\phi$. Theoretical properties about the BHEP test are given in Baringhaus and Henze [8], Csörgő [13], Henze and Zirkler [23], Henze [24] and Henze and Wagner [25]. A contextualization of the BHEP test approach in comparison with the most widely used test procedures can be found in Mecklin and Mundfrom [28] and Farrel et al. [19].

As noticed in some of the references listed above, the empirical power of the test based on $\mathbf{I}_{n}^{2}(h)$ varies considerably with the parameter $h$. In the univariate case, Epps and Pulley [15] considered the values $h=0.35,0.49,0.71,0.92$ and conclude that with $h=0.49$ or 0.71 the test is an omnibus normality test, whereas for $h=0.35$ and 0.92 the test seems to present a good performance against platykurtic and leptokurtic distributions, respectively. In the multivariate case Henze and Zirkler [23] conclude that for $h=1.41$ the test is powerful against heavy tailed distributions but they also report some extremely poor results for some other alternatives. However, after the initial papers about the BHEP test, it became a standard procedure to consider in practice the values $h=h_{\mathrm{EP}}:=0.71$ and $h=h_{\mathrm{HZ}}:=1.41$ for the univariate and multivariate cases, respectively, as we can see in the papers of Baringhaus et al. [9], Arcones and Wang [2], Mecklin and Mundfrom [28] and Farrel et al. [19].

Taking into account the fact that the formulation of the alternative hypothesis is in general impossible in a real situation, the main purpose of this paper is to examine the above-mentioned usual choices for the smoothing parameter $h$ and, if necessary, to propose alternative choices for $h$ that produce a generally powerful test against a wide range of alternatives. This question is particularly relevant in the multivariate case where the BHEP became a relevant test procedure that has been recommended in some recent comparative studies like those of Mecklin and Mundfrom [28] and Farrel et al. [19].

Although less interesting in practice we start our study by the analysis of the univariate case that we present in Section 3. This case will give us some important guidelines about the test behaviour as a function of the smoothing parameter $h$ that will have natural counterparts in a multivariate context. Contrary to the above-mentioned studies about the choice of $h$ that are exclusively based on Monte Carlo experiments, we begin our study by computing the Bahadur approximate indices of the univariate BHEP statistic for a set of Edgeworth alternatives. For that we use a result presented in Section 2 and proved in Section 5 about the Bahadur approximate slope of the BHEP statistic. These indices 
enable us to get a better understanding of the role played by the smoothing parameter in the detection of departures from the null hypothesis of normality in terms of each one of the third, fourth, fifth and sixth moments. They suggest that a large, but not too large, bandwidth is adequate for detecting departures from normality in skewness and kurtosis, whereas a small, but not too small, bandwidth is adequate for detecting high moment alternatives (see also Tenreiro [33] for the test of a simple hypothesis of normality). These conclusions agree with the finite sample power properties of the BHEP test that indicate that a small bandwidth should be used for short tailed alternatives whereas a large bandwidth is suitable for long tailed or skewed ones.

Taking into account the previous results, a Monte Carlo experiment based on a set of univariate alternative distributions which are members of the generalized lambda family was conducted in order to propose an easy-to-use rule for choosing the bandwidth. A similar simulation study is undertaken in Section 4 for the multivariate case $(2 \leq d \leq 15)$. Here we have considered a set of meta-Gaussian distributions whose marginal distributions are the univariate lambda distributions taken in the univariate case analysis.

Two distinct behaviour patterns were observed for the BHEP empirical power as functions of $h$ which led us to propose two distinct choices of the bandwidth, depending on the data dimension, which seem to present good performances against short tailed and long tailed alternative distributions. If we do not have relevant information about the tail of the alternative distributions, we propose to consider the mean value of the two previous bandwidths, $\bar{h}$, as rule-of-thumb for the choice of $h$. This way we intend to obtain a test with a reasonable performance against both types of alternatives.

In the univariate case our proposal is identical to the one by Epps and Pulley [15]. In the multidimensional case $\bar{h}$ behaves much better than $h_{\mathrm{HZ}}$ for short tailed alternatives and is only slightly inferior than $h_{\mathrm{Hz}}$ for long tailed alternatives. In practice, we strongly recommend the use of $\bar{h}$ that should replace, in a multivariate context, the usual choice of the bandwidth in the BHEP goodness-of-fit test. With this new bandwidth the BHEP test revealed a comparative good performance against a wide range of alternative distributions.

\section{Bahadur efficiency}

Denote by $\mathcal{D}$ the set of all bounded probability density functions $f$ on $\mathbb{R}^{d}$ with non-singular covariance matrix such that $\int\|x\|^{2} f(x) d x<+\infty$ and by $\mathcal{D}_{0}$ the subset of $\mathcal{D}$ of all densities that take the form (2) for some $m \in \mathbb{R}^{d}$ and some non-singular covariance matrix $S$. We are interested in the hypothesis testing problem $H_{0}: f \in \mathcal{D}_{0}$ versus $H_{1}: f \in \mathcal{D} \backslash \mathcal{D}_{0}$. In order to compare the tests based on $\mathbf{I}_{n}^{2}(h)$ given by (3) for different values of $h$, we give in 
this section the Bahadur [5] approximate slope $C_{\mathbf{I}(h)}$ of the so-called standard sequence of tests $\mathbf{I}(h)=\left(\mathbf{I}_{n}(h)\right)$, where $\mathbf{I}_{n}(h)=\mathbf{I}_{n}^{2}(h)^{1 / 2}$. For $f \in \mathcal{D} \backslash \mathcal{D}_{0}$, the Bahadur approximate slope is defined as the probability limit

$$
C_{\mathbf{I}(h)}(f):=-2 \lim _{n \rightarrow \infty} n^{-1} \log \left(1-F_{\infty, h}\left(\mathbf{I}_{n}(h)\right)\right),
$$

where $F_{\infty, h}$ is the asymptotic null cumulative distribution function of $\mathbf{I}_{n}(h)$ (see Tenreiro [34]). Taking into account that $1-F_{\infty, h}\left(\mathbf{I}_{n}(h)\right)$ is no more than the asymptotic $p$-value of $\mathbf{I}_{n}(h)$, given two different tests, the one with highest Bahadur approximate slope is preferred. For the description of Bahadur's concept of efficiency see Bahadur $[5,6,7]$ or Nikitin [29].

The next result follows from Gregory's [21] and Bahadur's [5] results. Its proof is given in Section 5.

Theorem 1. For $f \in \mathcal{D} \backslash \mathcal{D}_{0}$ we have

$$
C_{\mathbf{I}(h)}(f)=\lambda_{1, h}^{-1} b_{\mathbf{I}(h)}(f),
$$

where

$$
\begin{aligned}
b_{\mathbf{I}(h)}(f) & =\int\left|\phi_{h} \star f^{*}(x)-\phi_{h} \star \phi(x)\right|^{2} d x \\
& =(2 \pi)^{d} \int\left|\hat{f}^{*}(t)-\hat{\phi}(t)\right|^{2}|\hat{\phi}(h t)|^{2} d t,
\end{aligned}
$$

$f^{*}$ is the density in $\mathcal{D}$ defined by $f^{*}(x)=\left|S_{f}^{1 / 2}\right| f\left(m_{f}+S_{f}^{1 / 2} x\right)$, for $x \in \mathbb{R}^{d}$, where $m_{f}$ and $S_{f}$ are the mean and covariance matrix of $f$, and $\lambda_{1, h}$ is the largest eigenvalue of the symmetric positive semidefinite Hilbert-Schmidt operator $A_{h}$ defined, for $q \in L_{2}\left(\mathbb{R}^{d}, \mathcal{B}\left(\mathbb{R}^{d}\right), \Phi\right)=$ : $L_{2}(\Phi)$, by

$$
\left(A_{h} q\right)(u)=\int \mathcal{Q}(u, v ; h) q(v) d \Phi(v)
$$

where $\Phi=\phi \cdot \lambda$ with $\lambda$ the Lebesgue measure in $\mathcal{B}\left(\mathbb{R}^{d}\right)$ and $\mathcal{Q}(\cdot, \cdot ; \cdot)$ is the function defined, for $u, v \in \mathbb{R}^{d}$ and $h>0$, by

$$
\mathcal{Q}(u, v ; h)=\int \mathcal{K}(x, u ; h) \mathcal{K}(x, v ; h) d x
$$

where for $u=\left(u_{1}, \ldots, u_{d}\right), x=\left(x_{1}, \ldots, x_{d}\right) \in \mathbb{R}^{d}$ and $h>0$,

$$
\begin{aligned}
\mathcal{K}(x, u ; h)= & \phi_{h}(x-u)-\phi_{\left(1+h^{2}\right)^{1 / 2}}(x)+\sum_{p=1}^{d} \phi_{h} \star \psi_{p}(x) u_{p} \\
& +\frac{1}{2} \sum_{1 \leq p \leq q \leq d} \phi_{h} \star \psi_{p q}(x)\left(u_{p} u_{q}-\delta_{p q}\right),
\end{aligned}
$$

with $\psi_{p}(x)=-x_{p} \phi(x), \psi_{p q}(x)=\left(x_{p} x_{q}-\delta_{p q}\right) \phi(x)$ and $\delta_{p q}$ the Kronecker symbol. 
If the null density $\phi$ belongs to a family of probability density functions of the form $\{f(\cdot ; \theta): \theta \in \Theta\}$, where $\Theta$ is a non-trivial closed real interval containing the origin and $\phi=f(\cdot ; 0)$, it is natural to compare the set of competitor tests $\mathbf{I}^{2}(h), h>0$, through their Bahadur local approximate slopes $C_{\mathbf{I}(h)}(f(\cdot ; \theta))$ when $\theta \rightarrow 0$. Under some regularity conditions on the previous parametric family, two useful representations can be given for $C_{\mathbf{I}(h)}(f(\cdot ; \theta))$.

Assumptions on $\{f(\cdot ; \theta): \theta \in \Theta\}$

For all $\theta \in \Theta, f(\cdot ; \theta)$ has zero mean and identity covariance matrix, and for all $x \in \mathbb{R}^{d}$ the function $\theta \rightarrow f(x ; \theta)$ is continuously differentiable on $\Theta$, and there exists a neighbourhood of the origin $V \subset \Theta$ such that the function $x \rightarrow \sup _{\theta \in V}\left|\frac{\partial f}{\partial \theta}(x ; \theta)\right|$ is integrable on $\mathbb{R}^{d}$.

Under assumption $(\mathrm{P})$, we have

$$
C_{\mathbf{I}(h)}(f(\cdot ; \theta))=\lambda_{1, h}^{-1} \int\left|\phi_{h} \star \frac{\partial f}{\partial \theta}(\cdot ; 0)(x)\right|^{2} d x \theta^{2}(1+o(1)), \theta \rightarrow 0 .
$$

We conclude that the Bahadur local approximate slopes take the form $\theta^{2}(1+o(1))$, up to the multiplication by a constant, when $\theta \rightarrow 0$, and therefore for the comparison of tests $\mathbf{I}^{2}(h)$ it is sufficient to compare the coefficients of $\theta^{2}$ called approximate local indices.

An informative alternative representation can be given for the previous local approximate slope. Denote by $\left\{q_{k, h}, k \in \mathbb{N}_{0}\right\}$ the orthonormal basis of $L_{2}(\Phi)$ consisting of eigenfunctions of $A_{h}$, i.e., for all $k$ and $j, \int \mathcal{Q}(\cdot, v ; h) q_{k, h}(v) d \Phi(v)=\lambda_{k, h} q_{k, h}$, a.e. $(\Phi)$ and $\left\langle q_{k, h}, q_{j, h}\right\rangle=\delta_{k j}$, where $\langle\cdot, \cdot\rangle$ denotes the usual inner product in $L_{2}(\Phi)$. Under assumption $(\mathrm{P})$, if $\frac{\partial \ln f}{\partial \theta}(\cdot ; 0) \in L_{2}(\Phi)$ the approximate local slope $C_{\mathbf{I}(h)}(f(\cdot ; \theta))$ can be expressed in terms of the weights $\left(\lambda_{k, h}\right)$ and the principal components $\left(q_{k, h}\right)$ :

$$
C_{\mathbf{I}(h)}(f(\cdot ; \theta))=\sum_{k=1}^{\infty} \lambda_{1, h}^{-1} \lambda_{k, h} a_{k, h}^{2} \theta^{2}(1+o(1)), \theta \rightarrow 0,
$$

where

$$
a_{k, h}=\left\langle q_{k, h}, \frac{\partial \ln f}{\partial \theta}(\cdot ; 0)\right\rangle
$$

for $k=1,2, \ldots$

If the eigenvalue sequence $\left(\lambda_{k, h}\right)$ converges quickly to zero, it is clear from this representation that only a finite number of alternative directions effectively contribute to $C_{\mathbf{I}(h)}(f(\cdot ; \theta))$. As we will see in the next section, in the particular case of the BHEP statistic (4), the weights $\gamma_{k, h}=\lambda_{1, h}^{-1} \lambda_{k, h}$, for $k=2,3, \ldots$, strongly depend on the choice of $h$. 


\section{Testing a univariate hypothesis of normality}

In this section, in the particular case of testing a univariate hypothesis of normality, we use the results of the previous section to get a better understanding of the role played by the smoothing parameter in the detection of departures from the null hypothesis.

\subsection{Most significant weights}

As described in Section 2, the Bahadur approximate slope of the sequence $\left(\mathbf{I}_{n}^{2}(h)\right)$ depends on the weights $\left(\gamma_{k, h}\right)$, where $\gamma_{k, h}=\lambda_{1, h}^{-1} \lambda_{k, h}$, and on the principal components $\left(q_{k, h}\right)$. Numerical evaluations of the most significant weights are shown in Table 1 for four values of $h$. These approximations have been obtained along the lines described in Tenreiro [33].

From these values and representation (6) we expect that for small values of $h$ the test based on $\mathbf{I}_{n}^{2}(h)$ could use information contained in other components different from the first ones. This conclusion agrees with the asymptotic properties of the test based on the invariant Bickel-Rosenblatt statistic $\mathbf{I}_{n}^{2}\left(h_{n}\right)$ where $h_{n}$ tends to zero when $n$ goes to infinity (see $[10,34])$. For moderate or large values of $h, \mathbf{I}_{n}^{2}(h)$ might exclusively use information contained in the first components.

\begin{tabular}{lllll}
\hline & $h=0.01$ & $h=0.1$ & $h=1.0$ & $h=2.0$ \\
\hline$\gamma_{2, h}$ & $9.99 \times 10^{-1}$ & $9.77 \times 10^{-1}$ & $4.54 \times 10^{-1}$ & $1.75 \times 10^{-1}$ \\
$\gamma_{3, h}$ & $9.71 \times 10^{-1}$ & $7.34 \times 10^{-1}$ & $5.31 \times 10^{-2}$ & $7.20 \times 10^{-3}$ \\
$\gamma_{4, h}$ & $9.70 \times 10^{-1}$ & $7.11 \times 10^{-1}$ & $2.08 \times 10^{-2}$ & $1.09 \times 10^{-3}$ \\
$\gamma_{5, h}$ & $9.44 \times 10^{-1}$ & $5.43 \times 10^{-1}$ & $3.31 \times 10^{-3}$ & $6.31 \times 10^{-5}$ \\
$\gamma_{6, h}$ & $9.42 \times 10^{-1}$ & $5.22 \times 10^{-1}$ & $1.20 \times 10^{-3}$ & $8.75 \times 10^{-6}$ \\
$\gamma_{7, h}$ & $9.17 \times 10^{-1}$ & $4.03 \times 10^{-1}$ & $2.18 \times 10^{-4}$ & $5.91 \times 10^{-7}$ \\
$\gamma_{8, h}$ & $9.15 \times 10^{-1}$ & $3.86 \times 10^{-1}$ & $7.55 \times 10^{-5}$ & $7.74 \times 10^{-8}$ \\
$\gamma_{9, h}$ & $8.91 \times 10^{-1}$ & $3.00 \times 10^{-1}$ & $1.48 \times 10^{-5}$ & $5.69 \times 10^{-9}$ \\
$\gamma_{10, h}$ & $8.89 \times 10^{-1}$ & $2.86 \times 10^{-1}$ & $4.95 \times 10^{-6}$ & $7.15 \times 10^{-10}$ \\
$\gamma_{11, h}$ & $8.65 \times 10^{-1}$ & $2.24 \times 10^{-1}$ & $1.03 \times 10^{-6}$ & $5.50 \times 10^{-11}$ \\
$\gamma_{12, h}$ & $8.64 \times 10^{-1}$ & $2.12 \times 10^{-1}$ & $3.33 \times 10^{-7}$ & $6.57 \times 10^{-12}$ \\
\hline
\end{tabular}

Table 1: Numerical evaluation of the most significant weights $\gamma_{k, h}=\lambda_{1, h}^{-1} \lambda_{k, h}$ for $\mathbf{I}(h)$, with $h=0.01,0.1,1.0,2.0$.

\subsection{Approximate local indices}

Using representation (5), in the following we compute the approximate local indices of $\mathbf{I}^{2}(h)$ for a set of local alternatives based on the Edgeworth series for the density which express 
departures from the null hypothesis in the $j$ th moment (for the Edgeworth expansion see Hall [22] and the references therein). We assume that the local alternatives satisfy assumption $(\mathrm{P})$ introduced in Section 2 with $f=f_{j}$, and are such that

$$
(A . j) \quad \frac{\partial \ln f_{j}}{\partial \theta}(\cdot ; 0)=H_{j}(\cdot) / \sqrt{j !},
$$

for $j=3, \ldots, 6$, where $H_{j}$ is the $j$ th Hermite polynomial defined by

$$
\begin{aligned}
& H_{3}(x)=x^{3}-3 x \\
& H_{4}(x)=x^{4}-6 x^{2}+3 \\
& H_{5}(x)=x^{5}-10 x^{3}+15 x ; \\
& H_{6}(x)=x^{6}-15 x^{4}+45 x^{2}-15 .
\end{aligned}
$$

The skewness and kurtosis alternatives considered by Durbin et al. [14] satisfy (A.3) and (A.4), respectively.

The approximate local indices plotted in Figure 1, for $h \in[0.01,2]$, agree with the previous conclusions. It is clear that a large, but not too large, bandwidth is adequate for detecting departures from normality in skewness and kurtosis, whereas a small, but not too small, bandwidth is adequate for detecting high moment alternatives.

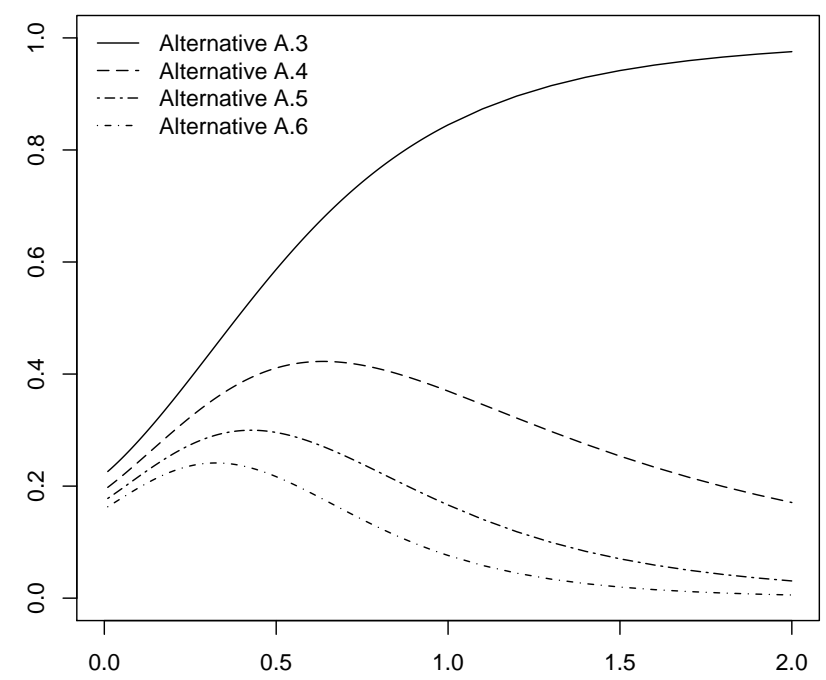

Figure 1: Approximate local indices for $\mathbf{I}(h), h \in[0.01,2]$

\subsection{The finite sample performance}

The main purposes of this section are to know if the finite sample power performance of the $\mathbf{I}^{2}(h)$ tests for fixed alternatives is in accordance with the theoretical properties based on Bahadur efficiency and also to provide some suggestions on the choice of $h$. 

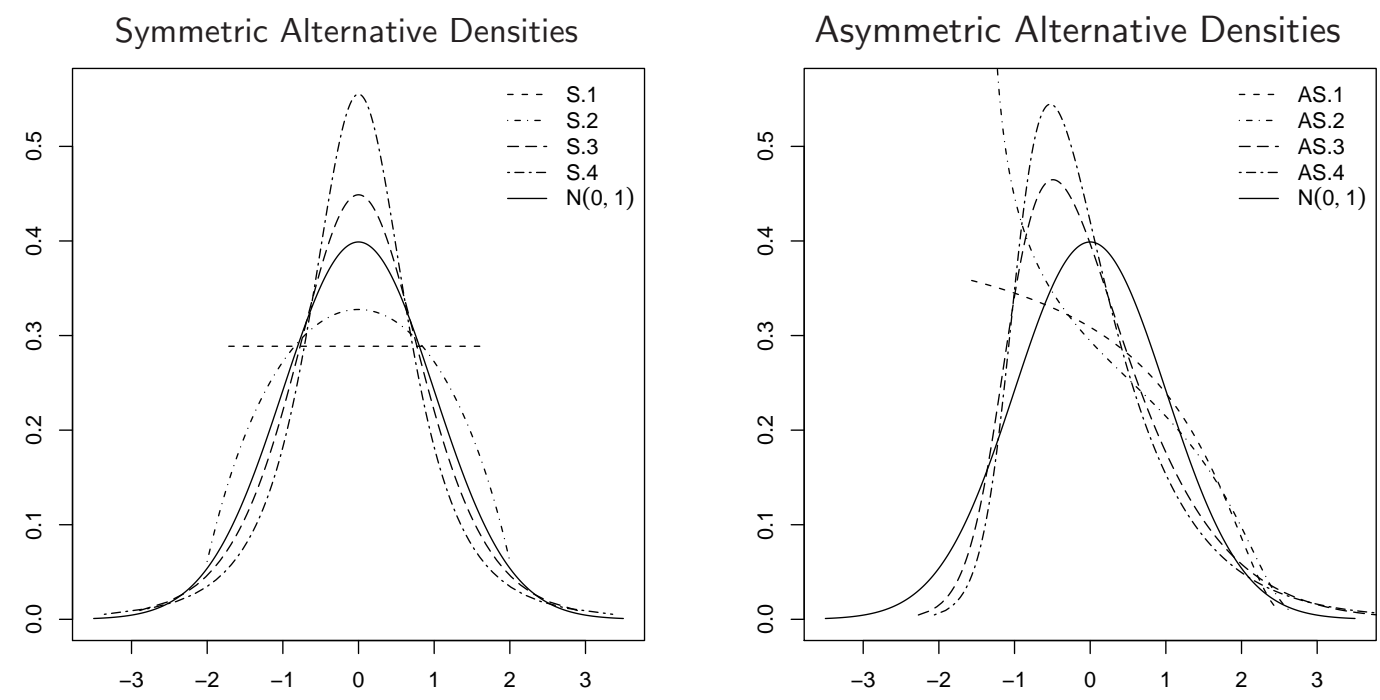

Figure 2: Densities used in the simulation study

In order to examine the performance of the $\mathbf{I}^{2}(h)$ tests for alternatives in skewness and kurtosis, we consider four symmetric and four asymmetric alternative distributions (four of them are platykurtic and the other four are leptokurtic) whose densities are shown in Figure 2. These distributions, which are members of the generalized lambda family discussed in Ramberg and Schmeiser [31], are easy to generate because they are defined in terms of the inverses of the cumulative distribution functions: $F^{-1}(u)=\lambda_{1}+\left(u^{\lambda_{3}}-(1-u)^{\lambda_{4}}\right) / \lambda_{2}$, for $0<u<1$. The parameters defining the distributions used in the study and the associated moments $\mu_{i}$ of orders three to six are given in Table 2. All the considered distributions have zero mean and unit variance. Some of these distributions are used in Fan [16] to examine the performance of the Bickel-Rosenblatt test with a bandwidth converging to zero as $n$ tends to infinity.

In Figure 3 we present the empirical power of $\mathbf{I}_{n}^{2}(h)$ at level 0.05 as a function of $h=0.05,(0.05), 2$ for some of the previous distributions and $n=40,60,80,100$. For the critical values evaluation we have used $10^{4}$ repetitions generated by the $\mathrm{R}$ function rnorm (cf. [30]) and the Monte Carlo power results are based on 2000 samples from the considered set of alternatives.

These empirical results are globally in accordance with the theoretical ones based on the Bahadur efficiency approach. For short tailed alternatives their departure from normality in skewness is necessarily small which explains the high power observed for small (but not too small) values of $h$ and the low power observed for large values of $h$. This is particularly true for symmetric alternatives. For long tailed (symmetric or asymmetric) alternatives a large value for $h$ should be chosen. As mentioned in the introductory section, these 


\begin{tabular}{|c|c|c|c|c|c|c|c|c|}
\hline Case & $\mu_{3}$ & $\mu_{4}$ & $\mu_{5}$ & $\mu_{6}$ & $\lambda_{1}$ & $\lambda_{2}$ & $\lambda_{3}$ & $\lambda_{4}$ \\
\hline & \multicolumn{8}{|c|}{ Symmetric distributions } \\
\hline S.1 & 0 & 1.80 & 0 & 3.86 & 0 & 0.577350 & 1 & 1 \\
\hline S.2 & 0 & 2.08 & 0 & 5.54 & 0 & 0.463251 & 0.5 & 0.5 \\
\hline S.3 & 0 & 4.06 & 0 & 35.90 & 0 & 0.017829 & 0.01 & 0.01 \\
\hline \multirow[t]{2}{*}{ S.4 } & 0 & 11.61 & 0 & 7211.95 & 0 & -0.397012 & -0.16 & -0.16 \\
\hline & \multicolumn{8}{|c|}{ Asymmetric distributions } \\
\hline AS.1 & 0.31 & 2.09 & 1.69 & 6.05 & 0.578020 & 0.465781 & 1 & 0.3 \\
\hline AS.2 & 0.51 & 2.22 & 2.74 & 7.40 & 0.835034 & 0.459063 & 1.4 & 0.25 \\
\hline AS.3 & 0.90 & 4.21 & 10.32 & 40.88 & -0.635145 & 0.096880 & 0.025 & 0.094 \\
\hline AS.4 & 1.52 & 7.46 & 33.83 & 209.04 & -0.628997 & -0.037156 & -0.0075 & -0.03 \\
\hline
\end{tabular}

Table 2: Distributions used in the simulation study

conclusions are also supported by the simulation studies undertaken by Epps and Pulley [15] and Baringhaus et al. [9].

\subsection{A rule-of-thumb for the choice of $h$}

In order to get an easy-to-use rule for choosing the bandwidth $h$, for each one of the previous alternative distributions and for sample sizes $n=20,40,60,80,100$, we compute the nearly optimal bandwidth $h_{f, n}$. This bandwidth is obtained from the bandwidth $h_{f, n}^{*}$ that maximizes the empirical power over the set $H=\{0.05,0.1,0.15, \ldots, 3.0\}$ of values of $h$ by taking $h_{f, n}=h_{f, n}^{*}$ for short tailed alternatives and $h_{f, n}=\inf \{h \in H: p(h)>$ $\left.0.995 p\left(h_{f, n}^{*}\right)\right\}$, where $p(h)$ denotes the empirical power of the BHEP test associated to the bandwidth $h$, for long tailed alternatives. This way we avoid very large bandwidths that do not lead to a significant power improvement (see distribution AS.4 in Figure 3).

We remark that $h_{f, n}^{*}$ does not depend significantly on the sample size $n$ but it strongly depends on the underlying alternative distribution tail type. The sample distribution of the bandwidths $h_{f, n}$ is described in Figure 4 for the BHEP test at level 0.05 for short tailed and long tailed alternatives, respectively.

As a rule-of-thumb for the choice of $h$ for these two types of underlying distributions we propose using the medians of the previous empirical distributions. For short tailed distributions we obtain $h_{\mathrm{S}}=0.45$, whereas for long tailed distributions we get $h_{\mathrm{L}}=0.975$. If we do not have relevant information about the alternative distributions, we propose using for smoothing parameter the mean value of the two previous bandwidths $\bar{h}=\left(h_{\mathrm{S}}+h_{\mathrm{L}}\right) / 2 \approx$ $0.71=h_{\mathrm{EP}}$. This proposal leads to the choice of $h$ suggested by Epps and Pulley [15] and 
S.2

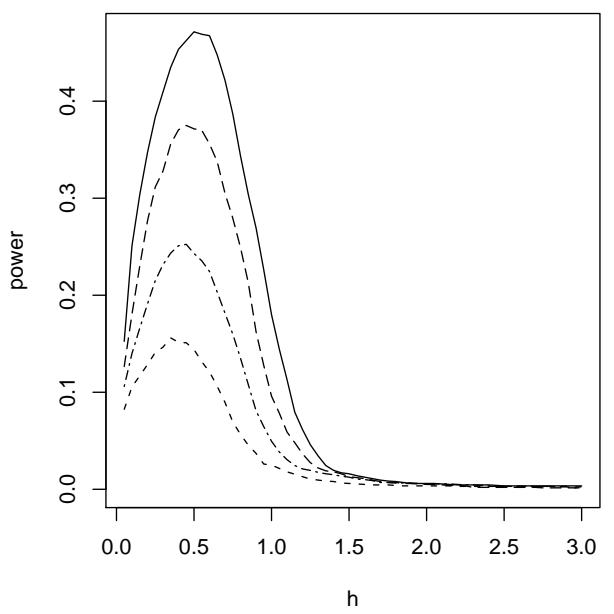

AS.2

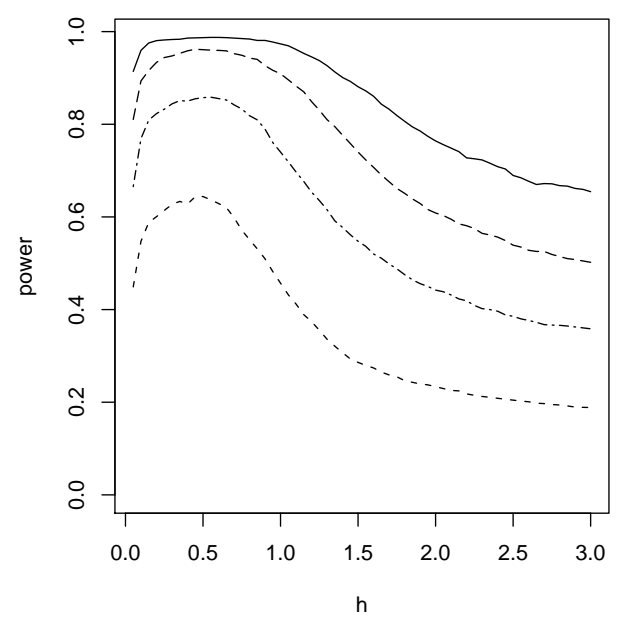

S.4

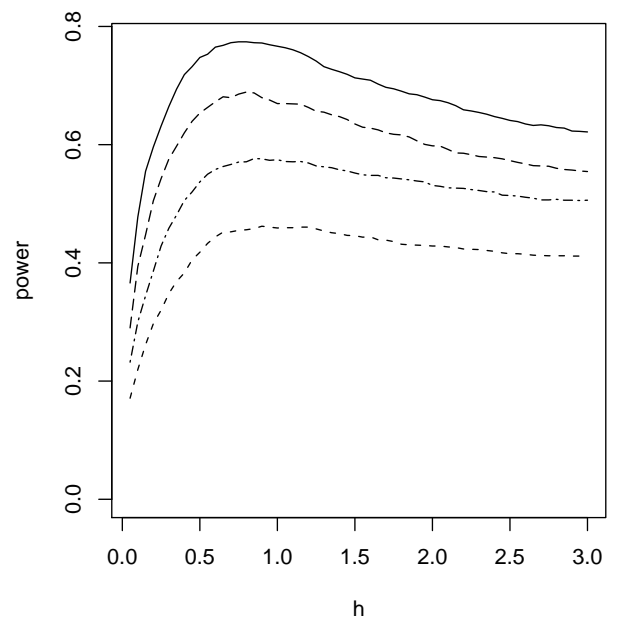

AS.3

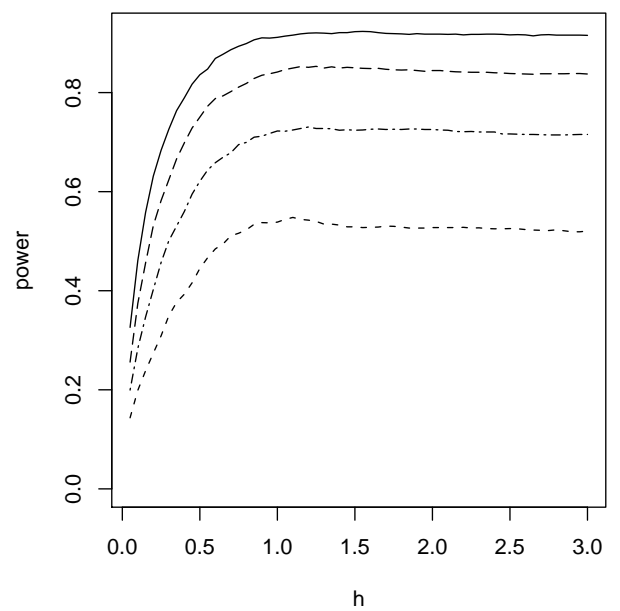

Figure 3: Empirical power as a function of $h$ for $n=100$ (solid line), $n=80$ (large broken line), $n=60$ (broken and dotted line), $n=40$ (short broken line), at level 0.05 .

also considered in other studies like those of Baringhaus et al. [9] and Arcones and Wang $[3]$.

\subsection{Finite sample power analysis}

Based on a simulation study undertaken for a large number of univariate alternative distributions usually considered in power studies for testing univariate normality, we conclude that the choices $h_{\mathrm{S}}$ and $h_{\mathrm{L}}$ present good performance for short tailed and high moment alternatives and for long tailed alternatives, respectively. Moreover, $\bar{h}$ presents a reasonable performance against both types of alternatives. These conclusions agree quite well with the results described in Epps and Pulley [15]. 


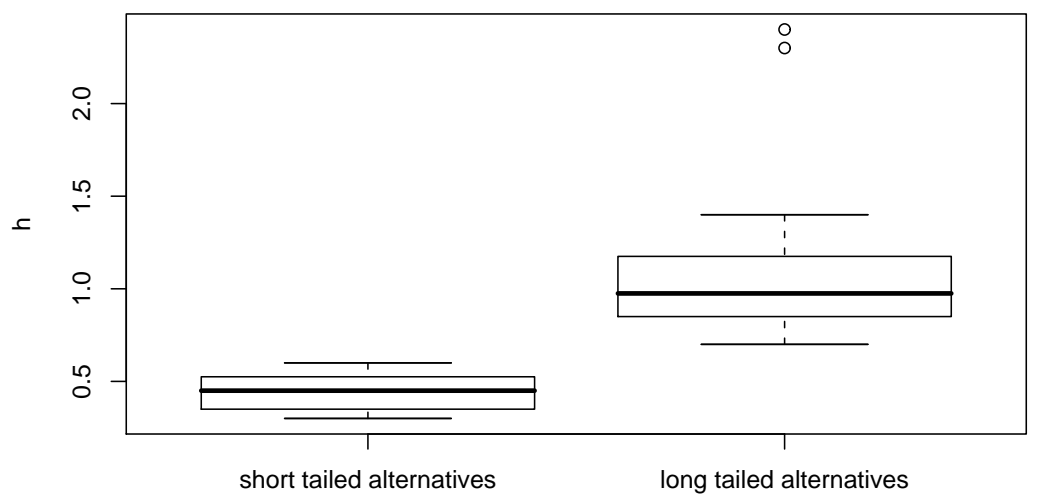

Figure 4: Distribution of $h_{f, n}$ at level 0.05

We limit ourselves to present in Figure 5 the empirical power results obtained for some of the considered alternatives. They give us a good picture about the overall observed power results. The uniform and beta distributions are short tailed whereas the Student and the lognormal are long tailed distributions (symmetric and asymmetric, respectively). The considered generalized exponential power distributions, GEP, whose distribution shape depends on two parameters, are high moment alternatives. They have zero mean, unit variance and their third and fourth order moments are equal to the normal distribution ones (see Johnson et al. [26] for the GEP distribution family).

The evaluation of the critical values of $\mathbf{I}_{n}^{2}(h)$ for $h=h_{\mathrm{S}}, h_{\mathrm{L}}, \bar{h}$ was based on $10^{5}$ repetitions under the null hypothesis and the power results were based on $10^{4}$ samples of different sizes from the considered set of alternatives. With $10^{4}$ repetitions, the margin of error for approximate $95 \%$ confidence intervals for the proportion of rejections does not exceed 0.01 .

Finally we remark that the previous rules-of-thumb can also be used for the BHEP test at levels 0.01 and 0.1 , since the distributions of the bandwidths $h_{f, n}$ for these two levels are similar to the ones shown in Figure 4.

\section{Testing a multivariate hypothesis of normality}

The test of a multivariate normality hypothesis is discussed in this section. Contrary to the univariate case, where theoretical and simulation based results have been used to study the test properties, the performance of the multivariate BHEP test is exclusively assessed through a Monte Carlo study.

However, it is natural to expect that the behaviour of the test $\mathbf{I}_{n}^{2}(h)$ as a function of $h$ described in the univariate case could have natural counterparts in a multivariate context. A first indication in this direction is given by the simulation study undertaken by Henze 

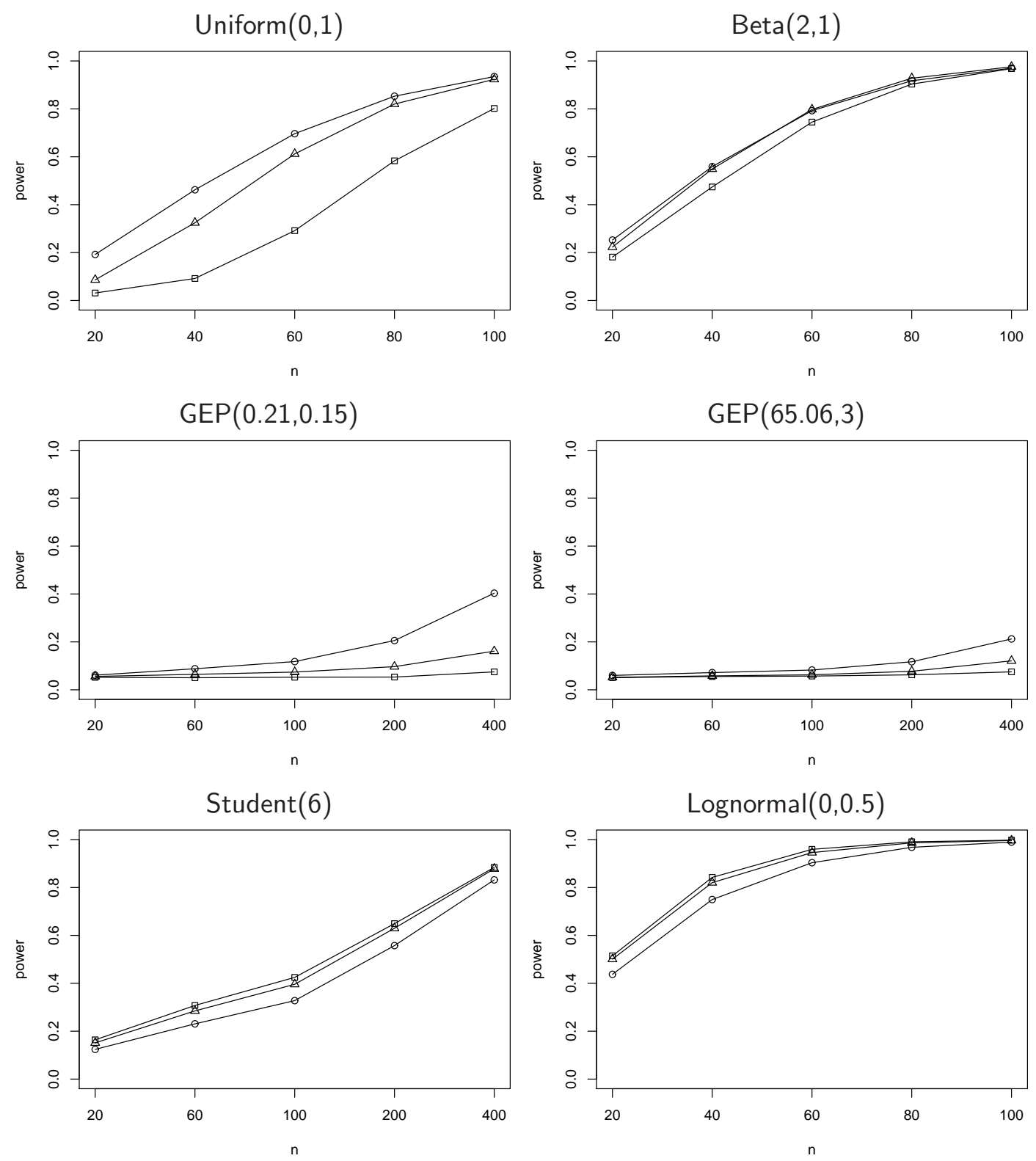

Figure 5: Empirical power for $h=h_{\mathrm{S}}(\bigcirc), h=h_{\mathrm{L}}(\square)$ and $h=\bar{h}(\triangle)$

and Zirkler [23] who considered the bandwidths $h=0.23,0.71,1.41$ and compared the associated tests with other procedures for assessing multivariate normality. The test with $h=0.23$ was generally inferior to the other two BHEP tests and the choice $h=1.41$ gave excellent results for asymmetric or long tailed distributions but it also showed a weak performance for symmetric and short tailed distributions. 


\subsection{A rule-of-thumb for the choice of $h$}

In order to propose a practical rule for the choice of the bandwidth, we conduct a simulation study based on a set of meta-Gaussian distributions whose marginal distributions are the univariate distributions considered in Section 3.3 (see Fang et al. [18] for the idea of metatype distribution). If $Z=\left(Z_{1}, \ldots, Z_{d}\right)$ is a multivariate normal distribution with mean zero and unitary diagonal covariance matrix $\Sigma$, and $F$ is the cumulative distribution function of one of the generalized lambda distributions S.1, ., AS.4, the alternative distributions we consider are the distributions of the vector $X=\left(X_{1}, \ldots, X_{d}\right)$, defined by $X_{i}=F^{-1}\left(\Phi\left(Z_{i}\right)\right)$, for $i=1, \ldots, d$, where $\Phi$ is the cumulative distribution function of the univariate standard normal distribution. Since we consider $\Sigma=\left[\sigma_{i j}\right]=\Sigma_{\rho}$ such that $\sigma_{i i}=1$ and $\sigma_{i j}=\rho$, with $0 \leq \rho<1$, for $1 \leq i \leq j \leq d$, the previous distributions will be denoted by S. $1_{d}(\rho), \ldots$, AS. $4_{d}(\rho)$.

After some simulation work it appears that the value of $h$ that maximizes the empirical power of the BHEP test does not depend significantly on the sample size $n$. However, it depends on the data dimension, and mainly on the underlying alternative distribution. Moreover, as in the univariate case, if we want a test with the omnibus property, extreme choices of $h$ have to be avoided. These facts are illustrated in Figure 6 where we present the two typical behaviours, for short tailed and long tailed alternatives, of the BHEP empirical power as a function of $h$, for $n=40,60,80,100, d=2,5,10$ and $h=0.05,(0.05), 3.0$. For the evaluation of the critical values we generated $10^{4}$ samples using the $\mathrm{R}$ function mvrnorm (cf. [30]), and the power estimates are based on 2000 samples from the considered set of alternatives.

In general, the bandwidth that maximizes the power seems to be an increasing function of the data dimension $d$. However, it is interesting to remark the large empirical power obtained for small values of $h$ for the short tailed distributions S. $1_{d}(\rho)$, S. $2_{d}(\rho)$, AS. $1_{d}(\rho)$, AS. $2_{d}(\rho)$ when $\rho$ is small and $d$ is large (see Figure 6 for distribution S. $1_{10}(0.3)$ ). This type of behaviour was for the first time pointed out by Henze and Wagner [25] for the uniform distribution over the unit $d$-cube and for a symmetric Pearson Type II distribution when $d=5$. A non-asymptotic argument can explain this behaviour. For a fixed $n$, from representation (4) we see that for large values of $d$ and depending on the sparseness of the observations, the term $n^{-1} \sum_{1 \leq i<j \leq n} \phi_{\left(2 h^{2}\right)^{1 / 2}}\left(Y_{i}-Y_{j}\right)$ loses its influence and the sum $\sum_{i=1}^{n} \phi_{\left(1+2 h^{2}\right)^{1 / 2}}\left(Y_{i}\right)$ determines the random behaviour of the test statistic $\mathbf{I}_{n}^{2}(h)$ for small values of $h$. Assuming a normal approximation for the previous sum, we conclude that the power function of the test for an alternative $f$ is an increasing function of $\sqrt{n}\left(\mathrm{E}_{\phi} \phi_{\left(1+2 h^{2}\right)^{1 / 2}}\left(Y_{1}\right)-\mathrm{E}_{f^{*}} \phi_{\left(1+2 h^{2}\right)^{1 / 2}}\left(Y_{1}\right)\right)$ and therefore the test detects alternatives $f$ satisfying $\int \phi_{\left(1+2 h^{2}\right)^{1 / 2}}(u) \phi(u) d u>\int \phi_{\left(1+2 h^{2}\right)^{1 / 2}}(u) f^{*}(u) d u$. This is in particular true for 

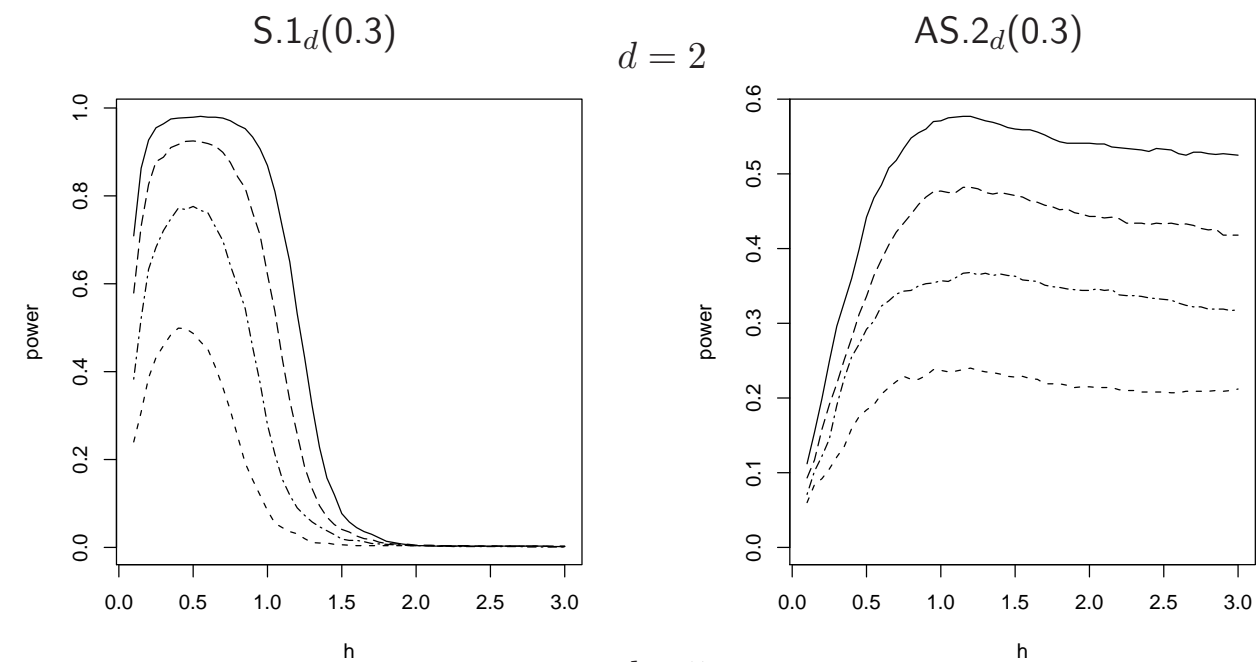

$d=5$
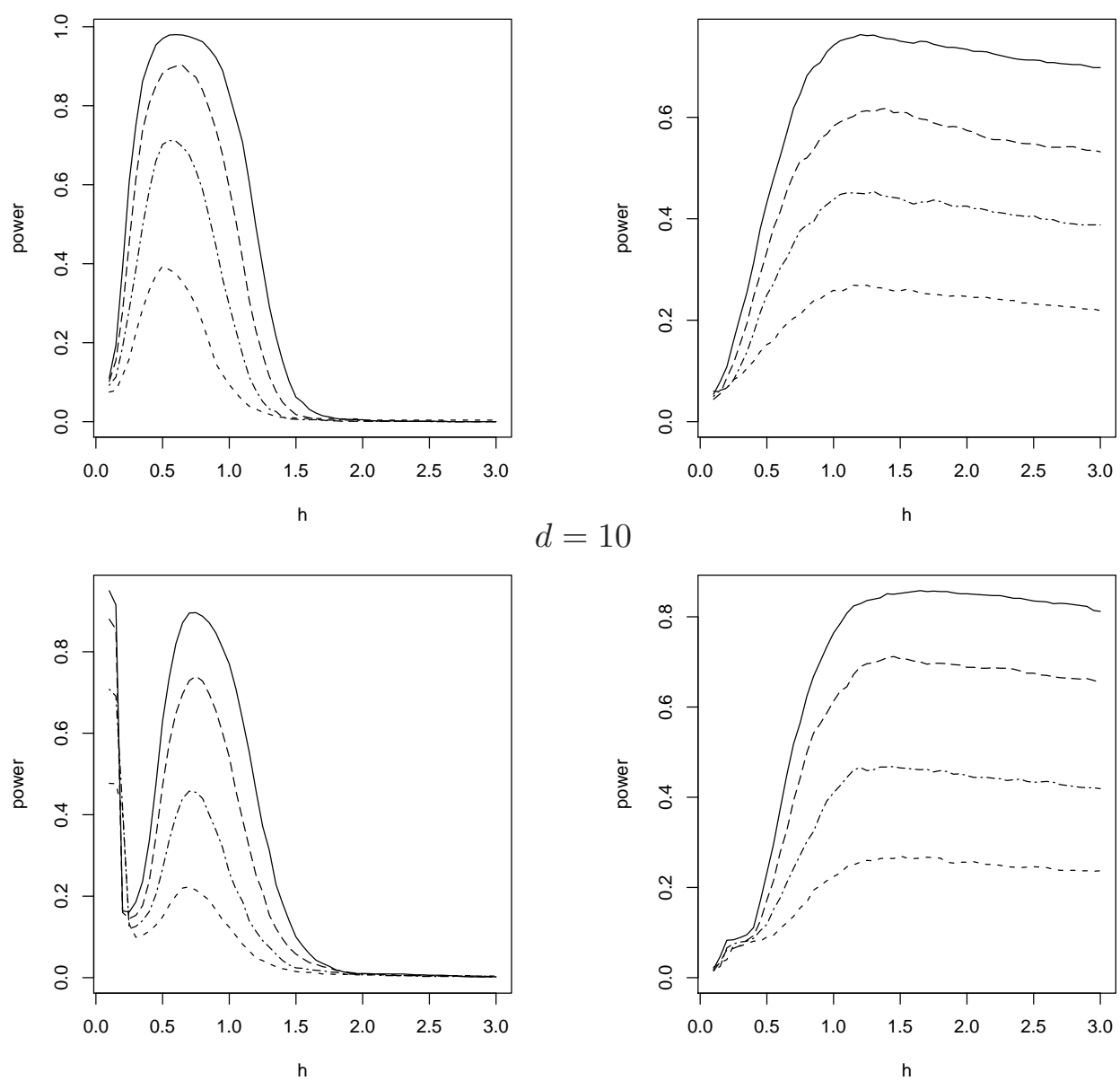

Figure 6: Empirical power as a function of $h$ for $n=100$ (solid line), $n=80$ (large broken line), $n=60$ (broken and dotted line), $n=40$ (short broken line), and $d=2,5,10$ at level 0.05 . 
short tailed distributions.

In order to get an easy-to-use rule for choosing the bandwidth, we proceed as in the univariate case. For data dimensions $d=2,(1), 10,12,15$, for each one of the alternative distributions S. $1_{d}(\rho), \ldots, \mathrm{AS} .4_{d}(\rho)$, where we take $\rho=0,0.3,0.7$, and sample sizes $n=$ $20,40,60,80,100$, we obtain the nearly optimal bandwidth $h_{f, n}$ defined in Section 3.4. However, when the empirical power presents the behaviour shown by distribution $\mathrm{S} .1_{10}(0.3)$ in Figure 6, the small values of $h$ that maximize the empirical power are not considered. In this case a large local maximum is taken for $h_{f, n}$.

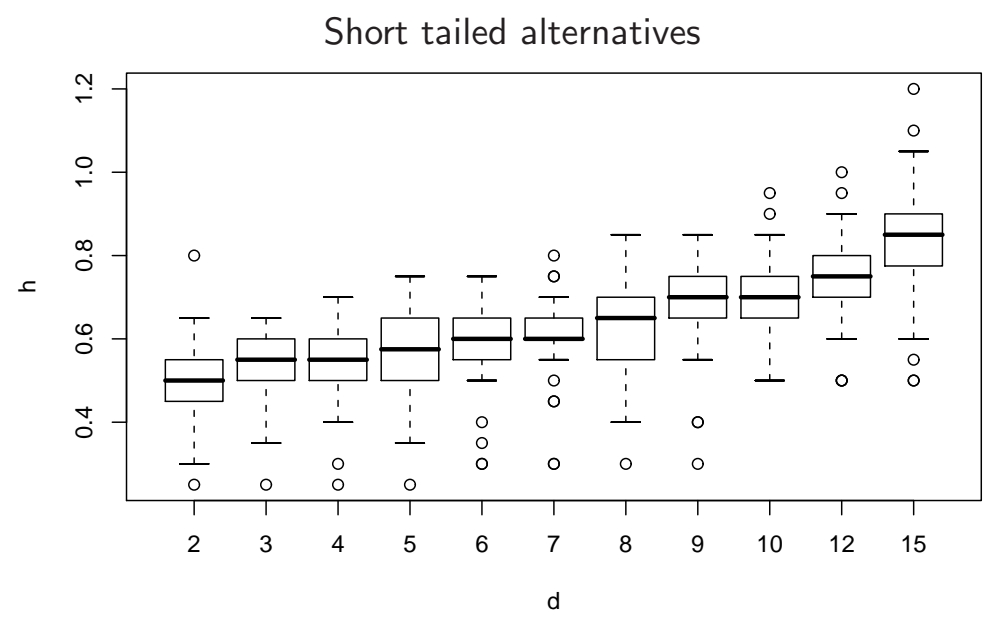

Long tailed alternatives

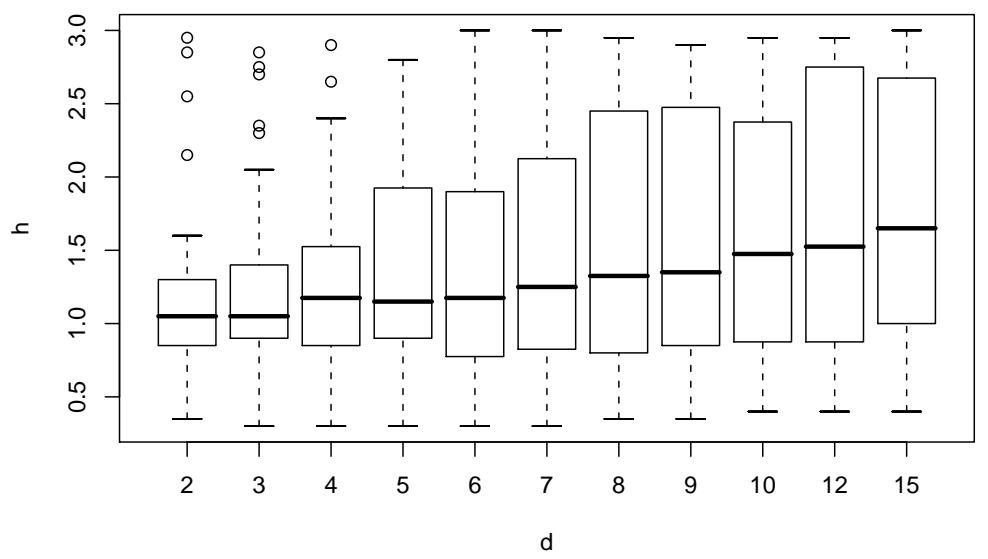

Figure 7: Distribution of $h_{f, n}$ as a function of $d$ at level 0.05

For each one of the considered data dimensions $d$, the sample distribution of the bandwidths $h_{f, n}$ for the BHEP test at level 0.05 is described in Figure 7 for short tailed and long tailed alternatives, respectively. A linear regression of the corresponding sample medians over the data dimension, leads to the following relation that we will use as rule-of-thumb for the choice of $h$ when the data dimension is $d$. For short tailed distributions we obtain 
$h_{\mathrm{S}}=0.448+0.026 d$, whereas for long tailed distributions the relation is $h_{\mathrm{L}}=0.928+0.049 d$. These rules-of-thumb can also be used for the BHEP test at levels 0.01 and 0.1 , since the distributions of the bandwidths $h_{f, n}$ for these two levels are very similar to the ones shown in Figure 7.

As in the univariate case, if we do not have relevant information about the tail of the alternative distribution, we propose taking $h=\bar{h}:=\left(h_{\mathrm{S}}+h_{\mathrm{L}}\right) / 2$. In Figure 8 these bandwidths are plotted as a function of $d$. Contrary to the usual choice $h_{\mathrm{HZ}}=1.41$ proposed by Henze and Zirkler [23] that seems to be more suitable for long tailed alternatives, we expect that $\bar{h}$ could produce a omnibus test of normality showing a reasonable performance against a large set of alternative distributions.

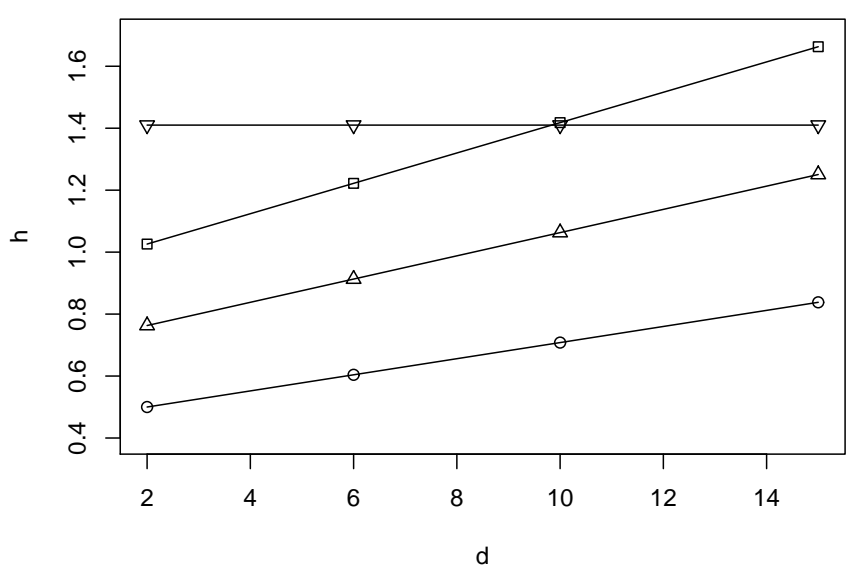

Figure 8: Considered choices of $h$ as functions of $d: h=h_{\mathrm{S}}(\bigcirc), h=h_{\mathrm{L}}(\square), h=\bar{h}(\triangle)$ and $h=h_{\mathrm{HZ}}(\nabla)$

\subsection{Finite sample power analysis}

A simulation was conducted to compare the power of the BHEP tests for the following choices of the smoothing parameter: $h=h_{\mathrm{S}}, h_{\mathrm{L}}, \bar{h}, h_{\mathrm{HZ}}$. The considered set of alternative distributions includes all the alternatives proposed by Mecklin and Mundfrom [28] and also some other alternatives previously considered in the simulation studies of Henze and Zirkler [23] and Romeu and Ozturk [32]. The Mecklin and Mundfrom set of distributions, also considered in Farrel et al. [19], includes a large variety of alternatives which are described in detail in [28]. We used the algorithms described in Johnson [27] to generate all the multivariate distributions. The evaluation of the test statistic critical values was based on $10^{5}$ repetitions under the null hypothesis and the power results are based on $10^{4}$ samples of different sizes from the considered set of alternatives. The standard level of significance 

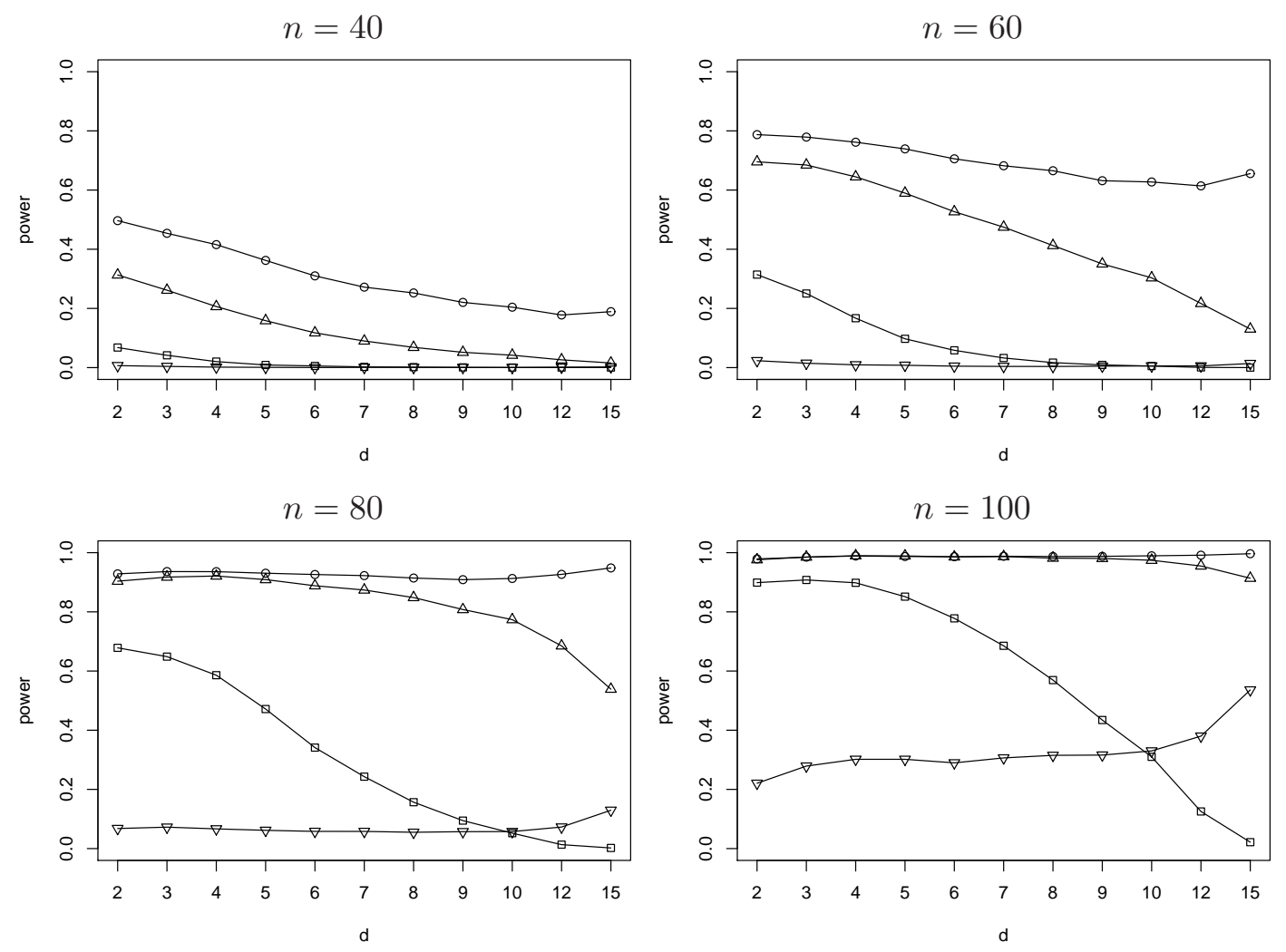

Figure 9: Empirical power for the multivariate uniform distribution from the Pearson Type II family for $h=h_{\mathrm{S}}(\bigcirc), h=h_{\mathrm{L}}(\square), h=\bar{h}(\triangle)$ and $h=h_{\mathrm{HZ}}(\nabla)$

$\alpha=0.05$ was used.

The pattern revealed by the tests in analysis was very clear: i) For short tailed distributions the best results were obtained by $h_{\mathrm{S}}$. The bandwidth $\bar{h}$ is better, and some times much better, than $h_{\mathrm{HZ}}$. In particular for low dimensions $d$ the test based on $h_{\mathrm{HZ}}$ reveals a poor performance against these alternatives. Figures 9 and 10 illustrate this situation showing the empirical powers for the Pearson Type II distributions with $m=0$ and $m=10$ (see [27] chapter 6). Contrary to the simulation results reported in Mecklin and Mundfrom [28] and Farrel al. [19] for the multivariate uniform distribution, the test based on $h_{\mathrm{HZ}}$ reveals a poor performance. This behaviour, which was also observed for all the considered members of the Pearson Type II family, agrees with the simulation results reported in Henze and Zirkler [23]. Similar to the univariate case, the previous behaviour was also observed for a high moment alternative like the Khintchine distribution with GEP marginals (see [27], chapter 8 and paragraph 2.4) as it is shown in Figure 11. This non-normal distribution presents an interesting departure from multivariate normality since the values of Mardia's skewness and kurtosis are equal to the multivariate normal distribution ones. ii) For long 

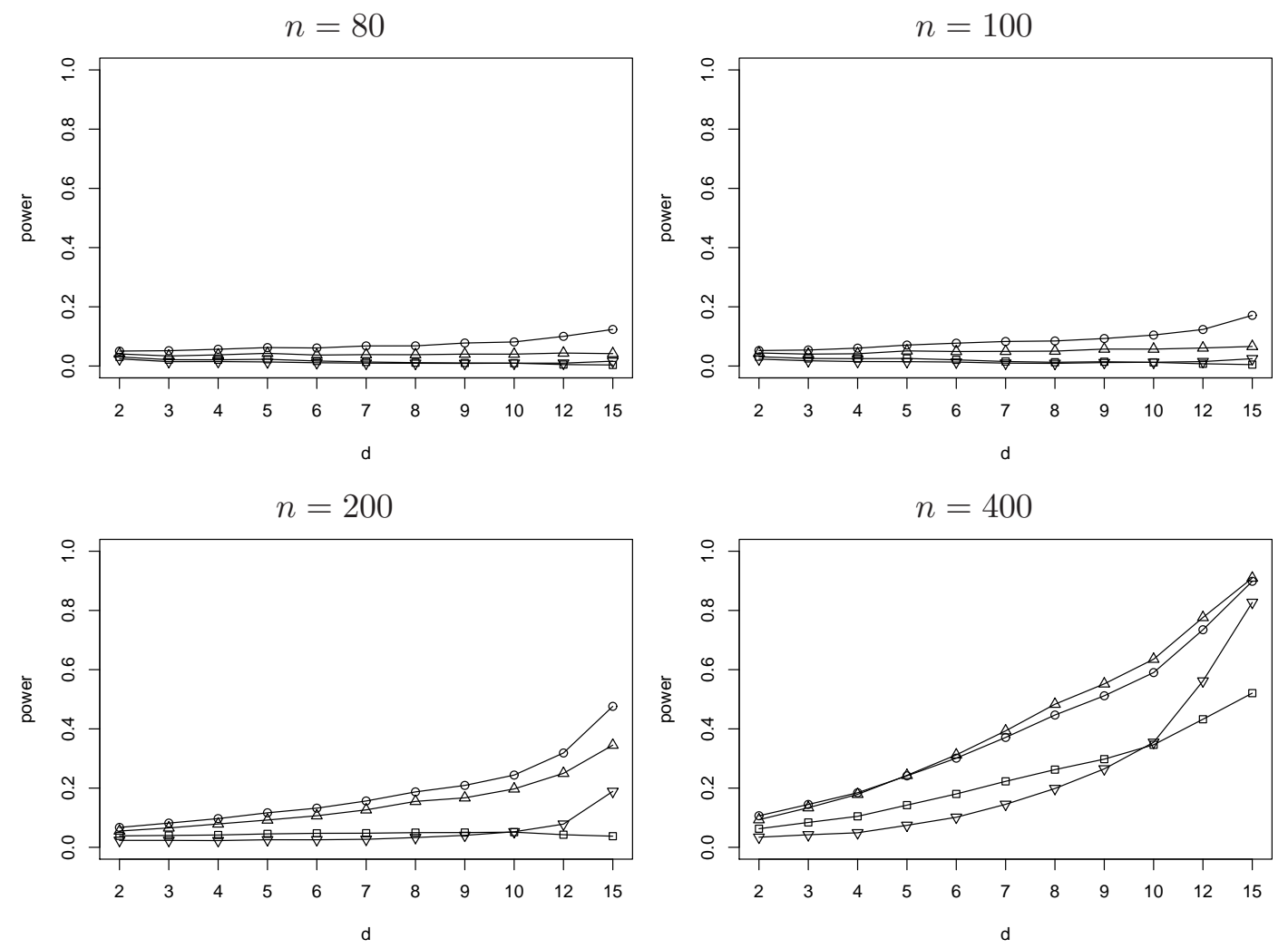

Figure 10: Empirical power for the Pearson Type II distribution with $m=10$ for $h=h_{\mathrm{S}}(\bigcirc)$, $h=h_{\mathrm{L}}(\square), h=\bar{h}(\triangle)$ and $h=h_{\mathrm{HZ}}(\nabla)$

tailed or moderately skewed alternatives the best results were obtained by the bandwidths $h_{\mathrm{L}}$ and $h_{\mathrm{HZ}}$. However, for large values of $d(d>10) h_{\mathrm{L}}$ is better than $h_{\mathrm{HZ}}$. Although inferior to $h_{\mathrm{HZ}}$, the bandwidth $\bar{h}$ revealed a reasonable performance against all the considered long tailed alternatives. Figures 12 and 13 present the power estimates for the multivariate Student distribution $t_{10}$ from the Pearson Type VII family (see [27] chapter 6) and for the asymmetric multivariate Burr-Pareto-Logistic distribution with normal marginals with parameter $\alpha=1$ (see [27] chapter 7). Finally, Figure 14 reports the power estimates for one of the fifteen different mixtures of two multivariate normals considered in Mecklin and Mundfrom [28] (normal mixture 13). This mixture reflects a mild contamination level and is skewed and leptokurtic.

\subsection{Conclusion}

The previous results show that the usual choice $h=h_{\mathrm{HZ}}$ gives the best results for long tailed or moderately skewed alternatives but it also produces very poor results for short tailed alternatives. In practice, if no relevant information about the tail of the alternatives 

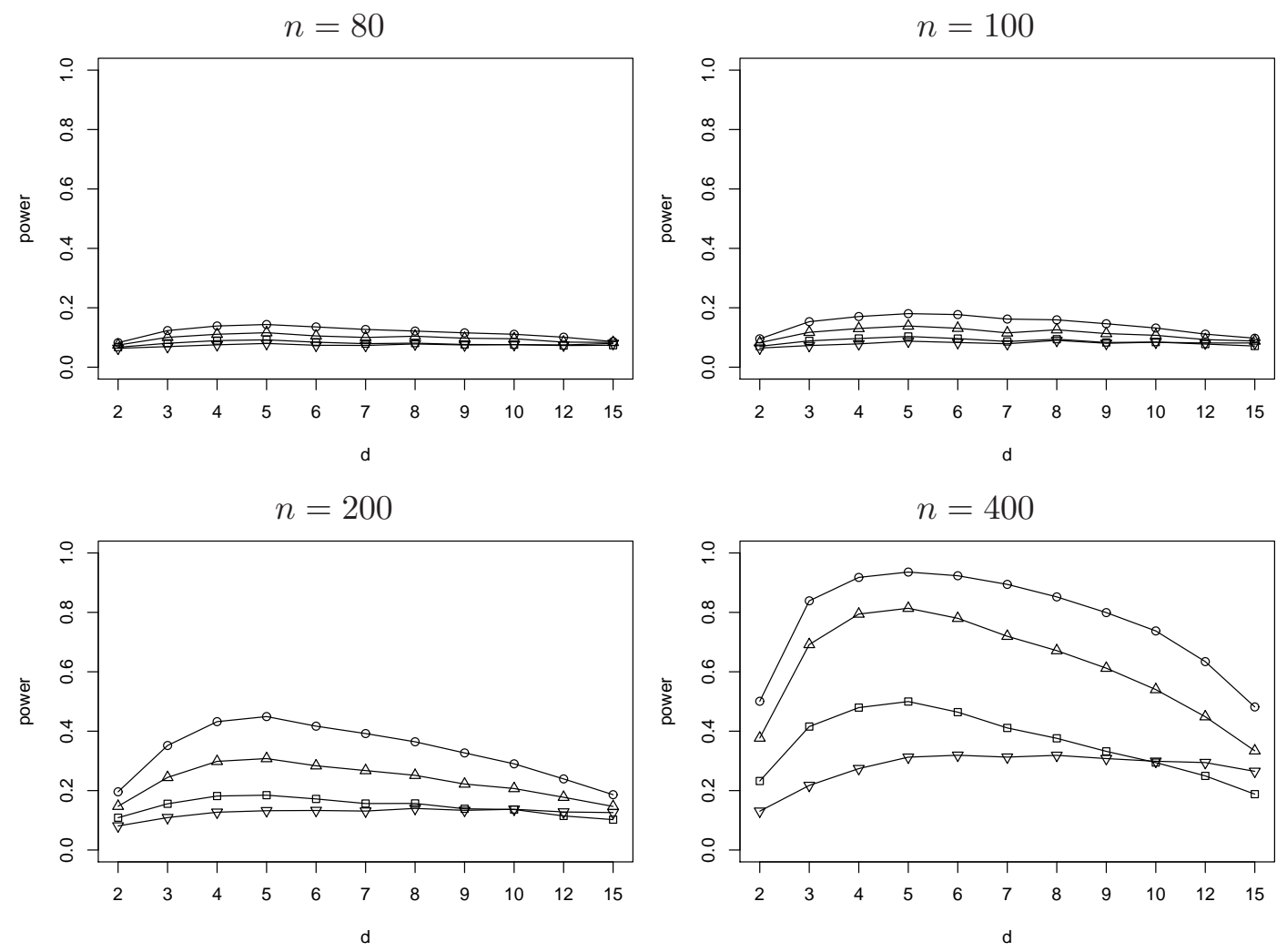

Figure 11: Empirical power for the high moment Khintchine distribution with GEP marginals for $h=h_{\mathrm{S}}(\bigcirc), h=h_{\mathrm{L}}(\square), h=\bar{h}(\triangle)$ and $h=h_{\mathrm{HZ}}(\nabla)$

is available, we strongly recommend the use of $\bar{h}$ that should replace, in a multivariate context, the usual choice of the bandwidth in the BHEP goodness-of-fit test. With this new bandwidth the BHEP test presents a reasonable performance against all the considered alternatives. Moreover, for short tailed alternatives and long tailed or moderately skewed alternatives the choices $h=h_{\mathrm{S}}$ and $h=h_{\mathrm{L}}$, respectively, are recommended.

\section{Proof of Theorem 1}

The following result follows straightforwardly from Lemma 2.4 of Gregory [21] and Bahadur's [5] results.

Lemma 1. Let $X_{1}, X_{2}, \ldots$ be a sequence of independent and identically distributed random variables whose distribution is determined by a parameter $\theta$ taking values in a set $\Theta$. Let $T=\left(T_{n}\right)$, with $T_{n}=T_{n}\left(X_{1}, \ldots, X_{n}\right) \geq 0$, be a sequence of test statistics to test $\theta \in \Theta_{0}$ against $\theta \in \Theta \backslash \Theta_{0}$, with $\Theta_{0} \subset \Theta$, that satisfies the following conditions: 

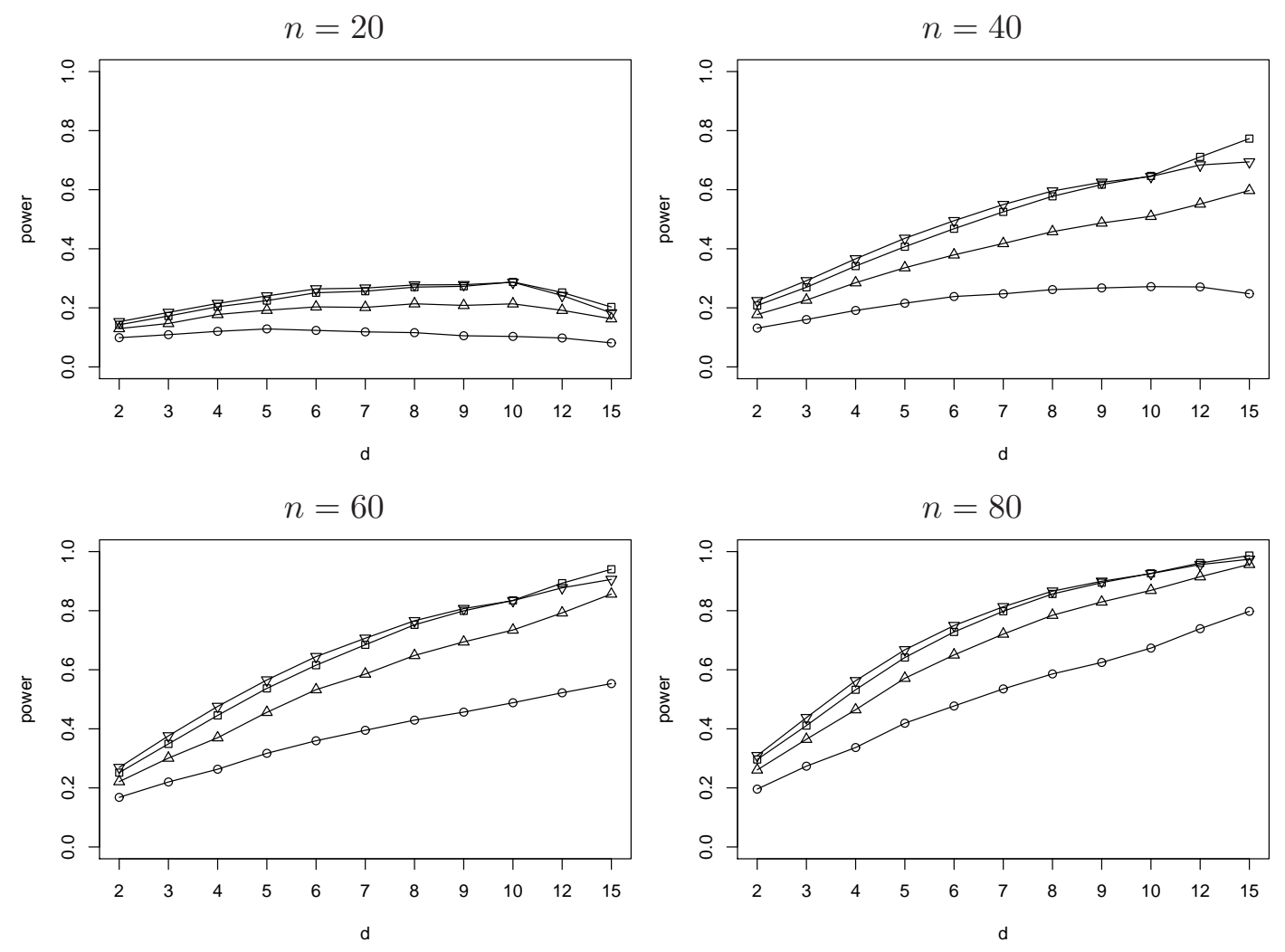

Figure 12: Empirical power for the multivariate Student distribution $t_{10}$ for $h=h_{\mathrm{S}}(\bigcirc)$, $h=h_{\mathrm{L}}(\square), h=\bar{h}(\triangle)$ and $h=h_{\mathrm{HZ}}(\nabla)$

A. For all $\theta \in \Theta_{0}$ we have

$$
T_{n}^{2} \stackrel{d}{\longrightarrow} \mu+\sum_{k=1}^{\infty} \lambda_{k}\left(Z_{k}^{2}-1\right),
$$

where $\mu \geq 0, \lambda_{k} \geq 0, k \in \mathbb{N}, \sum_{k=1}^{\infty} \lambda_{k}^{2}<\infty$, and $\left(Z_{k}\right)$ are iid standard normal random variables.

B. For all $\theta \in \Theta \backslash \Theta_{0}$,

$$
\frac{T_{n}^{2}}{n} \stackrel{p}{\longrightarrow} B(\theta)
$$

for some real and positive function $B$ on $\Theta \backslash \Theta_{0}$.

Then the Bahadur approximate slope of $T$ is given by

$$
C_{T}(\theta)=\lambda_{1}^{-1} B(\theta)
$$

for all $\theta \in \Theta \backslash \Theta_{0}$.

Taking in Lemma $1, \Theta=\mathcal{D}, \Theta_{0}=\mathcal{D}_{0}$ and $T_{n}=\mathbf{I}_{n}^{2}(h)^{1 / 2}$, Theorem 1 follows easily from 

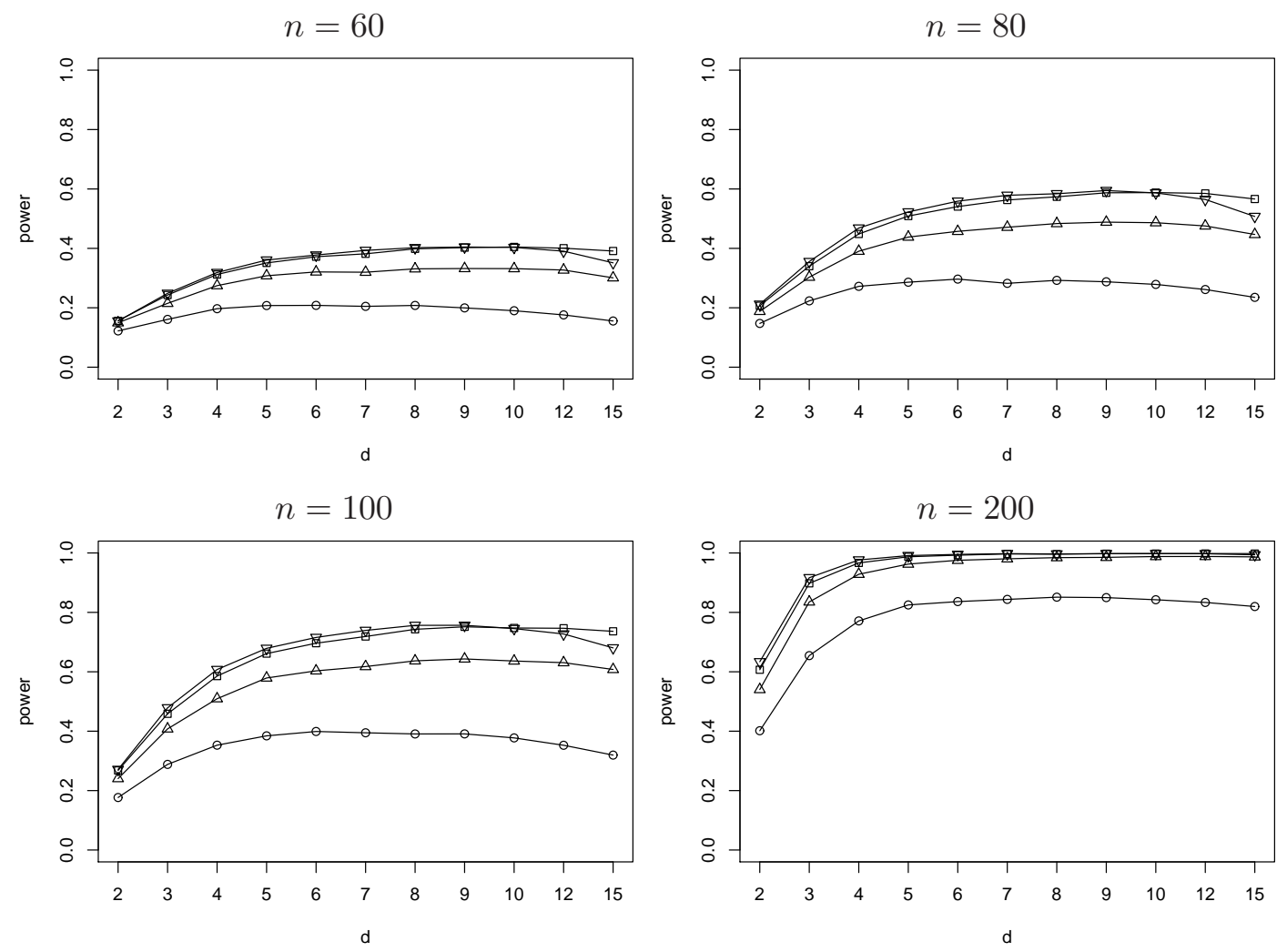

Figure 13: Empirical power for the multivariate Burr-Pareto-Logistic distribution with normal marginals with $\alpha=1$ for $h=h_{\mathrm{S}}(\bigcirc), h=h_{\mathrm{L}}(\square), h=\bar{h}(\triangle)$ and $h=h_{\mathrm{HZ}}(\nabla)$

Theorems 1 and 2 of Tenreiro [34] since conditions $\mathbf{A}$ and $\mathbf{B}$ are satisfied with

$$
\begin{gathered}
\mu=\int \mathcal{Q}(u, u ; h) d \Phi(u), \\
\lambda_{k}=\lambda_{k, h},
\end{gathered}
$$

and

$$
B(f)=(2 \pi)^{d} \int\left|\hat{f}^{*}(t)-\hat{\phi}(t)\right|^{2}|\hat{\phi}(h t)|^{2} d t
$$

\section{Acknowledgements}

The author expresses his thanks to the referees for their comments and suggestions. This research was partially supported by CMUC (Centre for Mathematics, University of Coimbra)/FCT. 

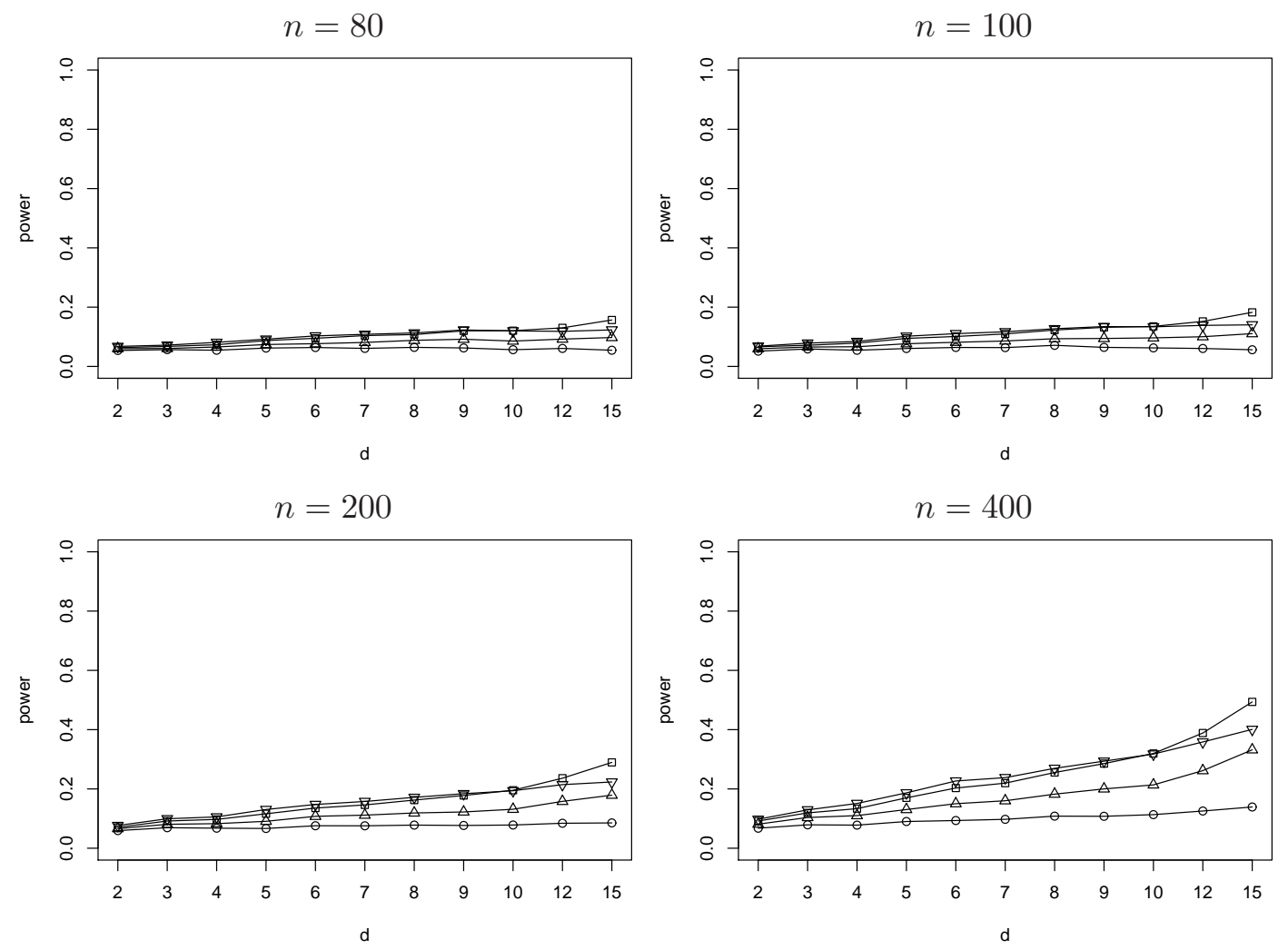

Figure 14: Empirical power for the mixture of normal distributions for $h=h_{\mathrm{S}}(\bigcirc), h=$ $h_{\mathrm{L}}(\square), h=\bar{h}(\triangle)$ and $h=h_{\mathrm{HZ}}(\nabla)$

\section{References}

[1] Anderson, N.H., Hall, P., Titterington, D.M. (1994). Two-sample test statistics for measuring discrepancies between two multivariate probability density functions using kernel-based density estimates. J. Multivariate Anal. 50, 41-54.

[2] Arcones, M.A. (2006). On the Bahadur slope of the Lilliefors and the Cramér-von Mises tests of normality. In: IMS Lecture Notes - High Dimensional Probability, Evarist Giné, Vladimir Koltchinskii, Wenbo Li, Joel Zinn, Eds., 51, 196-206.

[3] Arcones, M.A., Wang, Y. (2006) Some new tests for normality based on U-processes. Statis. Probab. Lett. 76, 69-82.

[4] Arcones, M.A. (2007). Two tests for multivariate normality based on the characteristic function. Math. Methods Statist. 16, 177-201.

[5] Bahadur, R.R. (1960). Stochastic comparison of tests. Ann. Math. Statist. 31, 276-295. 
[6] Bahadur, R.R. (1967). Rates of convergence of estimates and test statistics. Ann. Math. Statist. 38, 303-324.

[7] Bahadur, R.R. (1971). Some Limit Theorems in Statistics. Philadelphia, SIAM.

[8] Baringhaus, L., Henze, N. (1988). A consistent test for multivariate normality based on the empirical characteristic function. Metrika 35, 339-348.

[9] Baringhaus, L., Danschke, R., Henze, N. (1989). Recent and classical tests for normality. A comparative study. Comm. Statist. Simulation Comput. 18, 363-379.

[10] Bickel, P.J., Rosenblatt, M. (1973). On some global measures of the deviations of density function estimates. Ann. Statist. 1, 1071-1095.

[11] Bowman, A.W., Foster, P.J. (1993). Adaptive smoothing and density-based tests of multivariate normality. J. Amer. Statist. Assoc. 88, 529-537.

[12] Coin, D. (2008). A goodness-of-fit test for normality based on polynomial regression. Comput. Statist. Data Anal. 52, 2185-2198.

[13] Csörgö, S. (1989). Consistency of some tests for multivariate normality. Metrika 36, 107-116.

[14] Durbin, J., Knott, M., Taylor, C.C. (1975). Components of Cramér-von Mises Statistics, II. J. Roy. Statist. Soc. Ser. B 37, 216-237.

[15] Epps, T.W., Pulley, L.B. (1983). A test for normality based on the empirical characteristic function. Biometrika 70, 723-726.

[16] Fan, Y. (1994). Testing the goodness of fit of a parametric density function by kernel method. Econometric Theory 10, 316-356.

[17] Fan, Y. (1998). Goodness-of-fit tests based on kernel density estimators with fixed smoothing parameters. Econometric Theory 14, 604-621.

[18] Fang, H.-B., Fang, K.-T., Kotz, S. (2002). The meta-elliptical distributions with given marginals. J. Multivariate Anal. 82, 1-16.

[19] Farrel, P.J., Salibian-Barrera, M., Naczk, K. (2007). On tests for multivariate normality and associated simulation studies. J. Stat. Comput. Simul. 77, 1065-1080.

[20] Gouriéroux, C., Tenreiro, C. (2001). Local power properties of kernel based goodness of fit tests. J. Multivariate Anal. 78, 161-190. 
[21] Gregory, G.G. (1980). On efficiency and optimality of quadratic tests. Ann. Statist. 8, 116-131.

[22] Hall, P. (1997). The Bootstrap and Edgeworth Expansion. Springer, New York.

[23] Henze, N., Zirkler, B. (1990). A class of invariante consistent tests for multivariate normality. Comm. Stat. Theory Methods 19, 3595-3617.

[24] Henze, N. (1997). Extreme smoothing and testing for multivariate normality. Statist. Probab. Lett. 35, 203-213.

[25] Henze, N., Wagner, T. (1997). A new approach to the BHEP tests for multivariate normality. J. Multivariate Anal. 62, 1-23.

[26] Johnson, M.E., Tietjen, G.L., Beckman, R.J. (1980). A new family of probability distributions with applications to Monte Carlo studies. J. Amer. Statist. Assoc. 75, 276-279.

[27] Johnson, M.E. (1987). Multivariate Statistical Simulation. Wiley, New York.

[28] Mecklin, C.J., Mundfrom, D.J. (2005). A Monte Carlo comparison of Type I and Type II error rates of tests of multivariate normality. J. Stat. Comput. Simul. 75, 93-107.

[29] Nikitin, Y. (1995). Asymptotic efficiency of nonparametric tests. Cambridge University Press.

[30] R Development Core Team (2006). R: A Language and Environment for Statistical Computing. R Foundation for Statistical Computing, Vienna, Austria.

[31] Ramberg, J.S., Schmeiser, B.W. (1974). An approximate method for generating asymmetric random variables. Commun. ACM 17, 78-82.

[32] Romeu, J.L., Ozturk, A. (1993). A comparative study of goodness-of-fit tests for multivariate normality. J. Multivariate Anal. 46, 309-334.

[33] Tenreiro, C. (2005). On the role played by the fixed bandwidth in the Bickel-Rosenblatt goodness-of-fit test. SORT 29, 201-216.

[34] Tenreiro, C. (2007). On the asymptotic behaviour of location-scale invariant BickelRosenblatt tests. J. Statist. Plann. Inference 137, 103-116.

[35] Yazici, B., Yolacan, S. (2007). A comparison of various tests of normality J. Stat. Comput. Simul. 77, 175-183. 